\title{
A Mobility and Activity Integration System Supporting Sensitivity to Contexts in Dynamic Routing -Emotional MaaS-
}

\author{
Koichiro Kawashima ${ }^{\mathrm{a}, 1}$, Yasuhiro Hayashi ${ }^{\mathrm{b}}$, Yasushi Kiyoki ${ }^{\mathrm{b}}$, Tetsuya Mita ${ }^{\mathrm{a}, 2}$ \\ a JR East R\&D Center Frontier Service Laboratory \\ ${ }^{\mathrm{b}}$ Graduate School of Media and Governance, Keio University
}

\begin{abstract}
Dynamic routing with combinations of mobility and activity is expected as new methodology for supporting sensitivity to various contexts for traveling. It is important to realize the dynamics by integrating "mobility and activity" in physical and cyber spaces. This paper presents a mobility and activity integration system for making routing plans from an original point to a destination with a scenario as "sensitivity to context" on the route. The "sensitivity to context" expresses reactions to the intentions and situations of a moving user. This system applies semantic computing to find out the appropriate mobility and activity, that dynamically calculates semantic associations between user's intentions and mobility services. This system makes a moving plan reflecting "sensitivity to context" created by query creation operators for synthesizing and expressing "everyday intention" and "mobility situation." This system has a distance calculation function for "feature value vectors" expressing the means of mobility and the features of facility spots, and outputs some expected means of moving towards the destination with activities on the route.
\end{abstract}

Keywords. Semantic Computing, Mobility as a Service (MaaS), Sensitivity, Context, Query Creation Operator

\section{Introduction}

\subsection{Background}

One of the social issues identified in recent years is the concentration of the population in urban areas, and it is predicted that approximately $70 \%$ of the world's population will be concentrated in cities by 2050 , with a steady increase in large cities containing 10 million people or more [1]. A wide variety of issues arise from this over-concentration including environmental pollution, electricity/energy shortages, traffic jams, the spread of viral infections, and deterioration of regional areas. Smart cities are receiving attention as a mechanism of connecting and resolving these issues [2]. The concept of linking these issues was discussed in the UN summit of September 2015, and was included as an international goal in the SDGs from 2016 to 2030, which has been a common language for linking disparate ideas up to this point [3]. According to a survey by the Statistics Bureau of the MIAC (Table 1), there is a diverse range of forms for regional mobility in Japan, and many different issues are faced [4]. Therefore, mobility services that are suited

${ }^{1}$ Koichiro Kawashima, Tetsuya Mita, 2-479 Nisshincho, Kita-Ku, Saitama City, Saitama 331-8513, Japan; E-mail: \{kouichirou-kawashima,t-mita\}@jreast.co.jp

${ }^{2}$ Yasuhiro Hayashi, Yasushi Kiyoki, 5322 Endo, Fujisawa City, Kanagawa 252-0882, Japan; Email: yasuhiro.hayashi@keio.jp,kiyoki@sfc.keio.ac.jp 
to regional characteristics and transportation systems are expected.

Attempts at creating new mobile business formats that can resolve these issues include multimodal services for integrating, linking and optimizing the various means of mobility, sharing services for using them in a mutual way, demand-based transportation to match the behavior of mobile users, and a hybrid cargo/passenger service for transporting and carrying both cargo and passengers in an integrated way. Businesses and verification are rolled out in the various regions of Japan. Additionally, in order to cope with diversified mobility needs, micro mobility, green slow mobility, and mobility services with new characteristics, such as automated driving etc., are promoted and installed [5]. The spread of these mobilities will lead to realize smart cities.

Table 1 Regional mobility issues and mobility service business characteristics

\begin{tabular}{|c|c|c|c|}
\hline \multicolumn{2}{|c|}{ City type } & Summary & Examples of mobile tasks \\
\hline \multicolumn{2}{|c|}{$\begin{array}{c}\text { Large city } \\
\text { (Over } 500,000 \text { people) }\end{array}$} & $\begin{array}{c}\text { Ordinance-designated } \\
\text { cities, etc. }\end{array}$ & $\begin{array}{l}\text { - Daily road congestion and public transport congestion } \\
\text { - Cumbersome connectivity between travel modes } \\
\text { - Limited means of transportation in the last mile }\end{array}$ \\
\hline \multirow{2}{*}{$\begin{array}{c}\text { Medium- } \\
\text { sized city } \\
(50,000 \text { to } \\
500,000 \\
\text { people })\end{array}$} & \begin{tabular}{|c|} 
rivate car \\
sharing rate \\
Less than $50 \%$ \\
\end{tabular} & $\begin{array}{c}\text { Near a large city } \\
\text { Bedroom town, etc. }\end{array}$ & $\begin{array}{l}\text { - Limited means of transportation to the city center. } \\
\text { - Congestion of public transportation during commuting, etc. } \\
\text { - Mobility for the elderly in Old Town and other areas is an issue. }\end{array}$ \\
\hline & $\begin{array}{c}\text { rivate car } \\
\text { sharing rate } \\
\text { Over } 50 \%\end{array}$ & $\begin{array}{l}\text { The location of the local } \\
\text { prefectural office } \\
\text { Company town, etc. }\end{array}$ & $\begin{array}{l}\text { - Limited means of transportation other than private cars } \\
\text { - Decline in convenience and business potential of public transportation } \\
\text { - Elderly people face difficulties in securing transportation }\end{array}$ \\
\hline \multicolumn{2}{|c|}{$\begin{array}{l}\text { Suburbs / depopulated } \\
\text { areas } \\
(\mathbf{5 0 , 0 0 0} \text { people or less })\end{array}$} & Local suburbs, etc. & $\begin{array}{l}\text { - Transportation is mostly by private car, unable to maintain public } \\
\text { transportation. } \\
\text { - Elderly people face difficulties in securing transportation }\end{array}$ \\
\hline
\end{tabular}

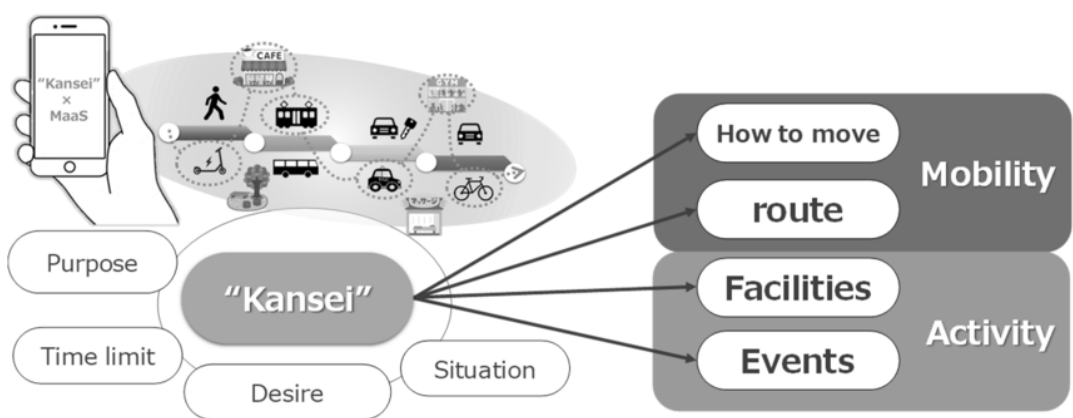

Figure 1 Image of Emotional MaaS

Mobility as a Service (MaaS) [6] is a concept of various mobility services integration as one service and people can perform a series of arrangements as one-stop service from search to payment. In other words, it means the spread of an integrated world of "physical space" and "information space" in mobility domain. One of the essential points of MaaS is that "people can choose the means for moving." Currently, people must make plans and search transports routes by themselves to have more convenience and drive cars by themselves, worrying if they'll make it in time. With autonomous cars and various mobile services, such as micro-mobility, multi-modality and sharing will be realized in our society, and people can release from the constraints and may spend most of daily time for creative and attractive works.

To express imagination, that is "sensitivity to movement with a dynamic context," we propose a mobility and activity integration system, named as Emotional MaaS, for computing sensitivity to movement in dynamic routing (Figure 1). This system makes routing plans for mobility and activity (facilities, stores, events etc.) from departure to destination with "sensitivity to movement with a dynamic context" expressing a user's intentions and mobility situation. 


\subsection{Related Studies}

\subsubsection{Semantic Computing}

Sensitivity to meaning, similarity, and association is dynamically changing according to contexts and situations in mobility and activity. It is important to apply semantic computing for selecting mobility and activity with a semantic computing model with dynamic context dependency. The semantic computing model [7] is used in a mobility and activity-selection process that dynamically supports sensitive equivalence, similarity, and association between mobile-user's intentions and existing services, according to "situation and context." This model is applied to realize the mobility and activity integration system for making routing plans with the sensitivity to contexts and situations for mobile users.

\subsubsection{Imagination-Based Image Query Creation Method Using Multiple Images}

An imagination-based image-query creation method has been proposed to create an image reflecting user's imagination with multiple images to express intentions on image search $[8,9]$. In this method, query creation operators and an image database for imagequery creation are defined in the front-end to an image database for search. The system applied the method creates an image-query reflecting an intention related to color and shape features according to the combination of several images and the operators, and searches images suited the intention. Using the concept of imagination-based imagequery creation method, an imagination-based query with a dynamic context is created to express situation and intentions for mobility and activity. To represent appropriate "mobility and activity", the context is represented the situation, that is named "mobility situation", as main purpose of moving and the intentions, that is named "everyday intention" as what a user wants to do during moving.

1.2.3 Creation of diverse driving travel plans that use the results of sensitivity assessments of people's motivation in traveling

It is promising to make travel plans that reflect sensitivity in tourism [10]. The motions for traveling have been classified into five elements, and the expected elements of each tourist is introduced. In this paper, we construct a sensitivity space using these five motive elements as a characterization of means of activity.

Table 2 Feature space structure for means of mobility

\begin{tabular}{|c|c|c|c|c|c|c|c|c|c|}
\hline Dimension & -1 & 0 & +1 & & Dimension & -1 & 0 & +1 & \\
\hline Price & low price & No relation & expensive & & Desk environment & & Not available & Available & \\
\hline Time required & Fast & No relation & Slow & $\ldots$ (a) & Communication environment & & Not available & Available & \\
\hline Crowding level & Low & No relation & High & & Exercise facilities & & Not available & Available & $\ldots(\mathrm{c})$ \\
\hline Regularity & High & No relation & Low & $\ldots$ (b) & View facilities & & Not available & Available & \\
\hline Motion/operation control & Unnecessary & No relation & Necessary & & Conversation space & & Not available & Available & \\
\hline
\end{tabular}

Table 3 Qualitative information feature correspondence table

\begin{tabular}{|c|c|c|c|c|c|}
\hline & \multicolumn{3}{|c|}{ Crowding Level } & \multirow{2}{*}{ Punctuality } & \multirow{2}{*}{$\begin{array}{c}\text { Operation and } \\
\text { Driving Maneuvers } \% 2\end{array}$} \\
\hline & Public Transportation & Walking & Uncongested Traffic $\% 1$ & & \\
\hline+1 & & & & & Awareness required \\
\hline+0.75 & 7:00 $<$ Moving time $<9: 00$ & & & Use public roads & \\
\hline+0.25 & $\begin{array}{c}18: 00<\text { movement time }< \\
21: 00\end{array}$ & & & $\begin{array}{c}\text { Use of independent track and } \\
\text { public road }\end{array}$ & \\
\hline 0 & $\begin{array}{c}\text { Travel time excluding rush } \\
\text { hours }\end{array}$ & & & $\begin{array}{l}\text { Self-propelled, such as on foot } \\
\text { or by bicycle }\end{array}$ & No awareness required \\
\hline-0.5 & & 0 & & & \\
\hline-1 & & & 0 & Use independent orbit & \\
\hline
\end{tabular}

*1 Mobility services that ensure private space and social distancing

$* 2$ If the required time (total mobility time) divided by the ratio of this mobility time is $t_{i}$, this can be calculated as $v_{i}\left(t_{i}\right)$ in previous (1). 


\section{Proposed Method}

\subsection{Feature Space Generation}

A feature space that supports the feature vector for means of "mobility and activity" is generated based on the procedures shown in the following steps 1-3.

Step 1: $n$ features in horizontal axis $\left(f_{1}, f_{2}, \ldots, f_{n}\right)$

Step 2: $m$ means of mobility or activity in the vertical axis $\left(O_{1}, O_{2}, \ldots, O_{m}\right)$

Examples of means of mobility: walking, regular route buses, railways, taxis

Examples of activity: favorite cafes, parks where you can exercise

Step 3: Apply metadata based on groups corresponding to the various means of mobility or activity.

\subsubsection{Means of mobility feature value vector expression}

The structure of the feature space for means of mobility is shown in Table 2.

(a) Definition of "price, required time, exercise consumption" from which quantitative information can be obtained

If there are $m$ target means of mobility for arbitrary interval

$$
\begin{gathered}
\text { Actual measured value vector } A_{\text {column }}=\left(a_{1}, a_{2}, \ldots, a_{m}\right) \\
\text { Feature value vector } V_{\text {column }}=\left(v_{1}, v_{2}, \ldots, v_{m}\right)
\end{gathered}
$$

At this time, feature vector elements $v_{i}$ for each means of mobility are defined as the expression (1). Each element is normalized in a non-linear way. This can be expressed as the feature value based on the market of an arbitrary interval. Further, by setting a threshold value, the impact of the outliers in the relative calculation can be minimized.

$$
\begin{aligned}
v_{i}\left(a_{i}\right):=\frac{a_{i}-\mu_{a}}{\sigma_{a}}\left(-1 \leqq v_{i}\right. & \leqq 1) \\
\text { Mean value } \mu_{a} & =\frac{1}{m} \sum_{i=1}^{m} a_{i} \quad \text { S. } D \sigma_{a}=\sqrt{\frac{1}{m} \sum_{i=1}^{m}\left(a_{i}-\mu_{m}\right)^{2}}
\end{aligned}
$$

(b) Definition of "human congestion, punctuality, action/operation manipulation" vector data, based on qualitative information

This is defined in the feature correspondence table shown in Table 3.

(c) Definition of other \{desk environment, communication environment, charging facili ties, exercise facilities, observation facilities, conversation space, eating/drinking area, 1 uggage space \} vector data

Vector elements are indicated in Table 2 as 1 for "Yes" and 0 for "No".

\subsubsection{Means of activity feature value vector data expression}

From the related studies described in 1.3.3, we can see that according to a survey by the Public Relations Office of the Cabinet, there are the five activity goal factors set as objectives to be achieved through mobility, including trips taken by people: (1) desire to enrich knowledge, (2)desire for personal (mental/physical) growth, (3)desire to alleviate tension, (4)desire to do something fun, and (5)desire to deepen human relationships [11]. The structure of the feature value space for means of activities are shown in Table 4.

(d) Definition of activity goal factor vector data

The vector elements of each means of activity are set to 1 for "Satisfied", 0.5 for "Quite satisfied", or 0 for "Unrelated", using information from Google Maps[12], Jalan[13], and Trip Advisor[14] for reference. (c) is the same as in 2.1.1. 
Table 4 Feature space structure for means of Activity

\begin{tabular}{|c|c|c|c|}
\hline dimension & 0 & +1 & \\
\hline desire to enrich knowledge & No relation & satisfaction & \multirow{5}{*}{$\cdots(d)$} \\
\hline desire for personal (mentalphysical) growth & No relation & satisfaction & \\
\hline desire to alleviate tension & No relation & satisfaction & \\
\hline desire to do something fun & No relation & satisfaction & \\
\hline desire to deepen human relationships & No relation & satisfaction & \\
\hline Desk Environment & Not available & Available & \multirow{8}{*}{$\cdots(\mathrm{c})$} \\
\hline Communication environment & Not available & Available & \\
\hline Charging facilities & Not available & Available & \\
\hline Exercise facilities & Not available & Available & \\
\hline View facilities & Not available & Available & \\
\hline Conversation space & Not available & Available & \\
\hline Eating and drinking space & Not available & Available & \\
\hline Luggage space & Not available & Available & \\
\hline
\end{tabular}

Table 5 "Everyday intentions" and "mobility situation" items

\begin{tabular}{|c|c|}
\hline Intention & Situation \\
\hline \hline To focus and concentrate & Commuting to work or school \\
To relax & Returning home \\
To exercise & Outing (day trip) \\
Sightseeing & Travel (more than 2 days) \\
companionship & Other travel \\
\cline { 2 - 2 } No particular intention & \\
\hline
\end{tabular}

\subsection{Sensitivity to Context and Query Creation Operators}

The query creation in this system is executed as follows. When humans pass their days accompanied by mobility, if we categorize them into "intrinsic motivation" and "extrinsic motivation," the former can be replaced by "everyday intentions" to achieve an arbitrary goal, and the latter as "mobility situations" in which you are placed. For the queries we used a "sensitivity to context vector" that merges those two motivations.

"Everyday intentions" can be separated for desires in daily activities, whereas "mobility situations" can be separated for the goal of the mobility, and those are shown in Table 5.

To give some examples, queries can be expressed as "commuting to work or school for purpose of exercise", "coming home to relax", "going out to interact with friends" or "traveling for sightseeing".

The size of the elements in each vector are set to their related size corresponding to (a), (b), (c), (d) in Tables 2 and 4.

Here, if we set the elements of each vector to

$$
\begin{aligned}
& \text { Daily intentions (Intention) } I=\left(i_{1}, \quad i_{2}, \ldots, \quad i_{n}\right), \\
& \text { Movement situtations(Situation) } S=\left(\begin{array}{ll}
s_{1} & S_{2}, \ldots, s_{n}
\end{array}\right)
\end{aligned}
$$

The query creation operators can be defined as in the expression (2).

$$
\begin{array}{r}
\text { query }=F(S, I) \text { OR } F(I, S)=\left(q_{1}, q_{2}, \ldots, q_{n}\right)(2) \\
\qquad F(A, B), A=\left(a_{1}, a_{2}, \ldots, a_{n}\right), B=\left(b_{1}, b_{2}, \ldots, b_{n}\right)
\end{array}
$$

At this time,

$$
\begin{aligned}
& \text { IF } a_{k} \cdot b_{k} \geqq 0 \text { THEN } q_{k}=\max _{0 \leq \mathrm{k} \leq 1}\left\{\left|a_{k}\right|,\left|b_{k}\right|\right\} \\
& \text { ELSE } a_{k} \cdot b_{k}<0 \text { THEN } q_{k}=a_{k}
\end{aligned}
$$

The merged sensitivity to a context vector has the maximum feature values for everyday intentions and mobility situations. For (a) and (b) in Tables 2 and 4, in case the symbols are reversed, $a_{k}$, depending on the situation in which the user is placed, may be selected dynamically as prioritized $i_{k}$ or $s_{k}$.

\subsection{Distance Calculation}

We perform a distance calculation for the "feature value vector" for means of mobility and activity stored in the database in 2.1 and "sensitivity to context file" generated in 2.2. 
At this time, the relative quantities for the means of mobility and activity are calculated respectively based on the subspace selection from a query[15].

The relative calculation quantity corresponds to feature value weight as a vector size, and as this is calculated with consideration for the orientation of the vector at this time, the inner product is used, and this is defined as in the expression (3).

In an arbitrary service (service_ $i$ ) with means of mobility and activity, if

$$
\begin{gathered}
\text { Sensitivity context } V_{q u e r y}=\left(v_{q 1}, v_{q 2}, \ldots, v_{q n}\right) \\
\text { Service feature value } V_{\text {service }_{i}}=\left(v_{s 1}, v_{s 2}, \ldots, v_{s n}\right), \\
\text { Relative quantity } C_{\text {servic }_{i}}\left(V_{\text {query }}, V_{\text {service }_{i}}\right):=\sum_{j=1}^{n} v_{q j} v_{s j}
\end{gathered}
$$

\section{Implementation Method}

In this section, we demonstrate the implementation of this system. With the point of departure fixed at the Tohoku Shinkansen South transfer ticket gate of Tokyo Station, examples of 4 destinations in the last mile from Tokyo station (Suitengu, Tokyo Tower, Tsukiji Honganji, Nihon Budokan) were used. The means of mobility were set as the mobility service and mobility route by each destination. Figure 2 shows the example of means of mobility from Tokyo station to Suitengu, and Figure 3 shows the selection mechanism for the means of mobility and activity.

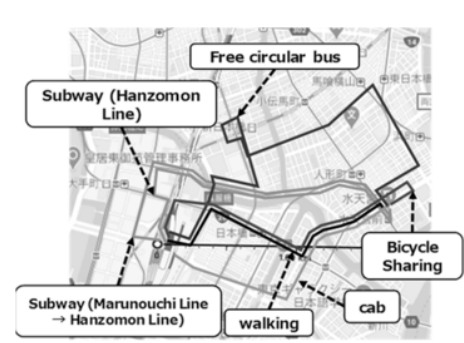

Figure 2 Means of mobility from Tokyo station to Suitengu

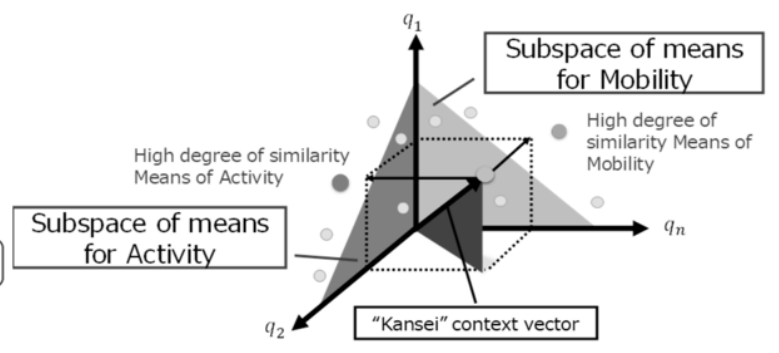

Figure 3 Means of mobility and activity subspace selection

\subsection{System Structure}

The system structure is as shown in Figure 4, and the flow of the execution process is indicated by Step 1 to 7 .

Step 1: Acquire input information from UI

Step 2: Generate query based on input information

Step 3: Filter means of mobility candidates based on input information (excess time), business hours, and weather information

Step 4: Perform distance calculation and select optimal solution for means of mobility

Step 5: Filter candidates for means of activity based on the means of mobility and business hours information from the optimal solution in Step 3

Step 6: Perform distance calculation and rank "facility spots" in mean of activity from 1 st to 5 th place

Step 7: Output combination of means of mobility and activity as information. 


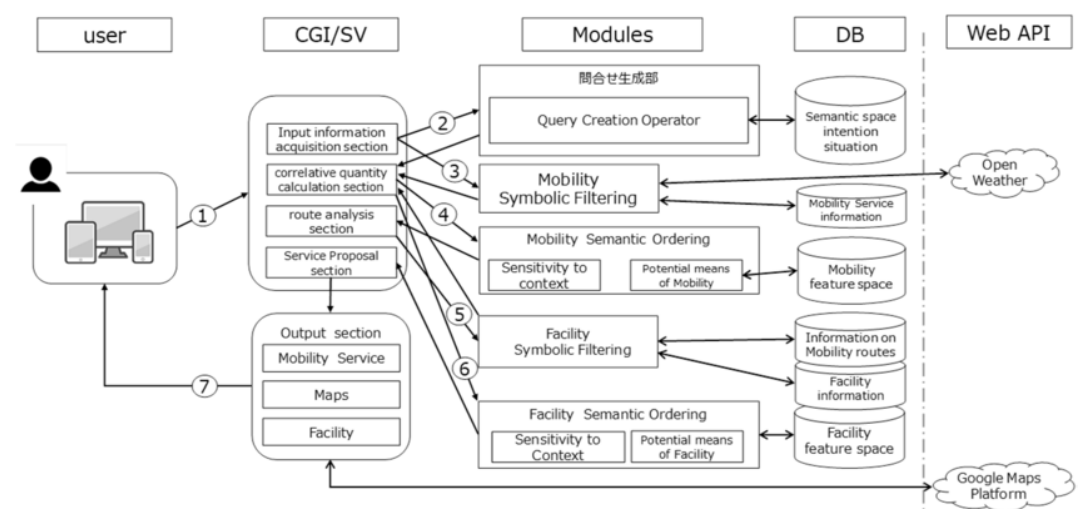

Figure 4 System Configuration Diagram

Figure 4 shows the objective of each filtering process. Filtering of the means of mobility in Step 3 prevents the return of unrealistic results to users (ex.: exclude transportation facilities outside of business hours and bicycle sharing not feasible on rainy days etc.) and lessens burden on the later processing section. In the same way, filtering of the facility spots in Step 5 sets points that are easy to stop by, and so are limited on the mobility routes in Step 4. Commonly, the mobility routes are connected in a straight line. By applying a fixed width to this straight line, this connects in a rectangle shape. By collating longitude and latitude information of a facility spot, this process can exclude facility spots outside of the rectangular area, in the Rectangle Mobility Scope Method (Figure 5). In the case of boarding-type mobility services (trains and buses), this process can be applied and limited to the stops on the mobile route, as the transport hub.

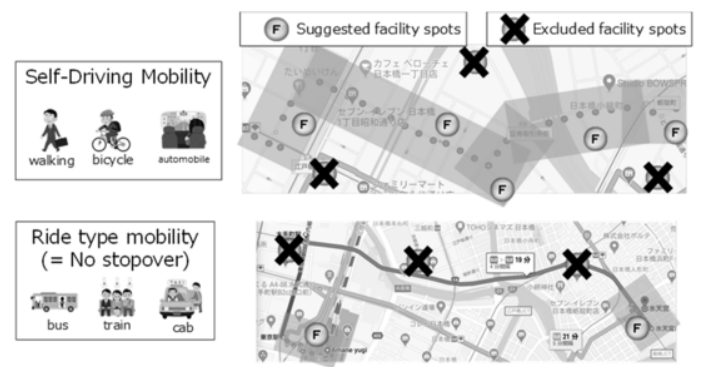

Figure 5 A Rectangle Mobility Scope Methed

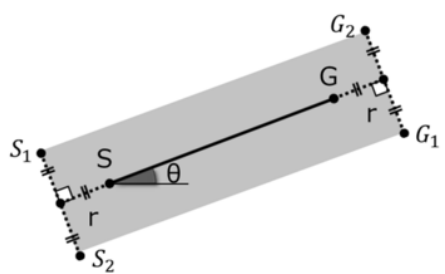

Figure 6 Acquisition of Rectangle Coordinates

The calculation method is defined as follows. It is treated as a plane orthogonal coordinate system. Let us set

$$
\begin{aligned}
& \text { Start coordinate within route } S=\left(\ln g_{1}, l a t_{1}\right) \\
& \text { End coordinate within route } G=\left(\operatorname{lng}_{2}, l a t_{2}\right)
\end{aligned}
$$

The Range is set to variable based on the selection of "everyday intention (Intention)".

$$
R[m]=\{\text { "intend to dedicate/concentrate": } 320 \text {, "intend to relax": } 240 \text {, }
$$

intend to exercise: 400 , intend to sightsee: 320 , intend to interact with friends: 320 , "no particular intention": 320$\}$

The distance at a longitude/latitude of $1^{\circ}$ around Tokyo Station is $90.4219[\mathrm{~km}]$, That is $r=\frac{R}{90421.9}$. The coordinate points in Figure 6 are 


$$
\begin{aligned}
& S_{1}=\left(l n g_{1}-r(\cos \theta+\sin \theta), l a t_{1}-r(\sin \theta-\cos \theta)\right) \\
& S_{2}=\left(l n g_{1}-r(\cos \theta-\sin \theta), l a t_{1}-r(\cos \theta+\sin \theta)\right) \\
& G_{1}=\left(l n g_{2}+r(\cos \theta+\sin \theta), l_{a t}+r(\cos \theta-\sin \theta)\right) \\
& G_{2}=\left(l n g_{2}+r(\cos \theta-\sin \theta), l a t_{2}+r(\cos \theta+\sin \theta)\right) \\
& \theta=\tan ^{-1}\left(\frac{l a t_{2}-l a t_{1}}{\operatorname{lng} g_{2}-\ln g_{1}}\right)
\end{aligned}
$$
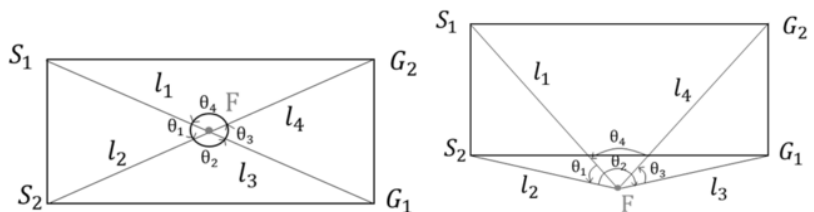

Figure 7 Internal/external judgement

Next, we judge the inside and outside of the facility spot points. With the rectangle at the peak $\left[S_{1}, S_{2}, G_{1}, G_{2}\right]$, when we can set an arbitrary facility point to $F=$ $\left(\operatorname{lng}_{f}, l a t_{f}\right)$, the side made with $F$ is set to $\left(l_{1} \ldots l_{4}\right)$. At this time, with the declination in Figure 7 as $\theta_{i}$, number of rotations $w n$ is defined as in the expression (4).

$$
w n:=\frac{1}{2 \pi} \sum_{i=0}^{3} \theta_{i}
$$

However, with regard to the orientation of declination $\theta_{i}$, if the respective cross products

$C_{1}=\overrightarrow{\mathrm{FS}} \mathrm{F}_{1} \times \overrightarrow{\mathrm{FS}}, C_{2}=\overrightarrow{F S_{2}} \times \overrightarrow{F G_{1}}, C_{3}=\overrightarrow{F G_{1}} \times \overrightarrow{F G_{2}}, C_{4}=\overrightarrow{F G_{2}} \times \overrightarrow{F S_{1}}$ are negative, the signals are treated as reverse rotations. Therefore,

$I F|w n|>0$, judged to be inner side ... true

$I F|w n|=0$, judged to be outer side ... false

\subsection{Used Programming Languages/Libraries}

Programming languages to be used for this system implementation are Python 3.8.2 and PostgreSQL 12.2. Used Python libraries are flask, psycopg2, numpy, pandas, matplotlib, scipy, googletrans, json. And for the display section, HTML, CSS, JavaScript and jQuery are utilized. Also, OpenWeatherMap to get weather information at an area [16], JapanHoliday to get public holidays announced by the Cabinet [17], and Google Maps Platform [18] to utilize a map and route information, are used as Web API.

\subsection{Input/Output Data}

The input values are "everyday intentions", "mobility situations", "time restrictions", and "destination", and commands issued with these. "Everyday intentions" and "mobility situations" are selected from a list. Additionally, for "time restrictions", when the arrival time is specified, the list is filtered down to means of mobility that allow you to arrive on time. "Destination" is selected from the map.

The optimal mobility service and mobility route to the destination is returned as map and text information. Additionally, the recommended facility spots that exist on the mobility route are returned in ranking format corresponding to the values in the expression (3), as map and text information. The input screen and output screen are as shown in Figure 8. 

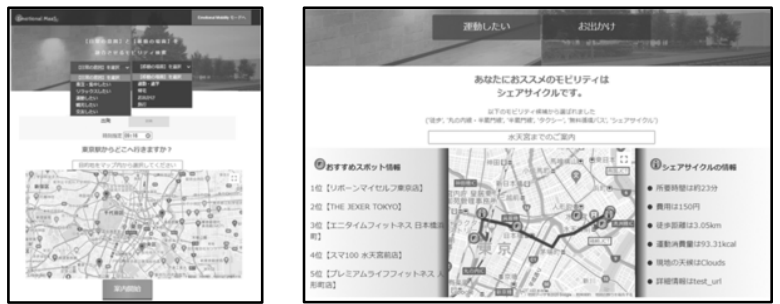

Figure 8 Input screen (left) and output screen (right)

\section{Evaluation}

In this section, we show the results and observations from three evaluation tests conducted with the objective for verifying the effectiveness of this method and system.

\subsection{Coverage rate of search targets}

This system measures the coverage rate of the search targets in relation to expressed sensitivity to context (generated queries and selected subspace). The aim of this verification is to confirm, in relation to the queries that can be expressed in this system, whether there is any polarization in the search targets. It also conforms its appropriateness by using a test collection, in the evaluations in 4.2 and 4.3 below. The coverage rate is as defined in the expression (5).

$$
\begin{aligned}
& \text { Coverage rate (Coverage) }:=\frac{\text { Conformity }}{q} \\
& q: \text { Number of queries that can be } \\
& \text { expressed }(29 \text { in this system) }
\end{aligned}
$$

Table 6 Coverage rate in the data space

\begin{tabular}{|c||c|c|c|c|c|}
\hline Destination & $\begin{array}{c}\text { Suitengu } \\
\text { Shrine }\end{array}$ & $\begin{array}{c}\text { Tokyo } \\
\text { Tower }\end{array}$ & $\begin{array}{c}\text { Tsukiji } \\
\text { Honganji } \\
\text { Temple }\end{array}$ & Budokan & Average \\
\hline Mobility & 1.000 & 0.966 & 0.966 & 1.000 & $\mathbf{0 . 9 8 3}$ \\
\hline Facility1st & 1.000 & 1.000 & 1.000 & 1.000 & $\mathbf{1 . 0 0 0}$ \\
\hline Facility2st & 1.000 & 1.000 & 1.000 & 0.966 & $\mathbf{0 . 9 9 1}$ \\
\hline Facility3st & 0.931 & 1.000 & 0.966 & 1.000 & $\mathbf{0 . 9 7 4}$ \\
\hline Facility4st & 0.931 & 0.966 & 0.931 & 0.966 & $\mathbf{0 . 9 4 8}$ \\
\hline Facility5st & 0.897 & 0.759 & 1.000 & 0.828 & $\mathbf{0 . 8 7 1}$ \\
\hline Facility Total & 0.952 & 0.945 & 0.979 & 0.952 & $\mathbf{0 . 9 5 7}$ \\
\hline
\end{tabular}

Table 7 "Everyday intention items (left) and "mobility scenario" question items (right)

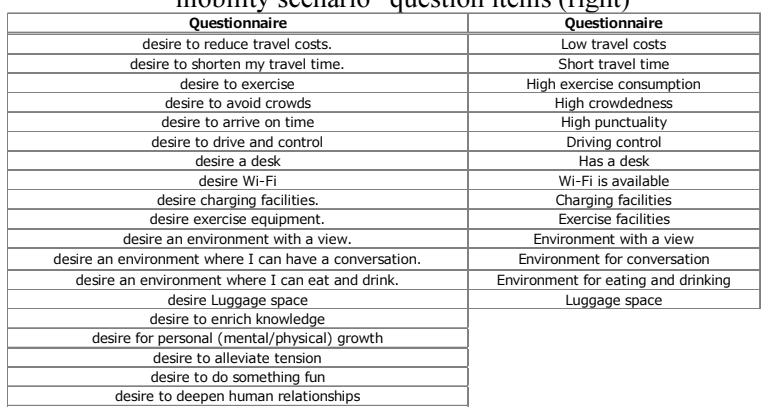

The coverage rate and data space distribution for each destination are shown in Table 6 . This indicator, when performing the correlation amount calculation, is used to confirm that the destination data with a high degree of similarity is output.

\subsection{Fill-rate for queries}

This system generates queries expressing the sensitivity to context of mobile users. We referred to a questionnaire survey for making queries representing user sensitivity. The questionnaire included respective items (Table 7) for "everyday intentions" and "mobility situations", and for the vector data, the ordinary data of (a), (b), (c), (d) in Tables 2, 4 were measured. The average value for 34 samples is shown in Figures 9, 10. 
The question items for intentions were answered with a 4-stage rating level \{think so, somewhat think so, don't really think so, don't think so , and the situation question items were answered with the 4 levels of \{applies, somewhat applies, does not really apply, does not apply\}.

\subsection{Fill rate in regard to the output value}

We have set up the appropriateness of the calculation method in the expressions (2) and (3), with regard to the search results in this system. This method was used 3 times for each person by 4 user test subjects, and a comparison between the query and output values was rated, as shown in Table 8 . Each query, the name of means for mobility, and facility spot genres 1-5 are displayed in Table 9. A comparative evaluation in relation to these search results is shown in Figures 11, 12, and 13.

Table 9 Queries and search results during experiment

\begin{tabular}{|c|c|c|c|c|c|c|c|c|c|c|c|c|}
\hline \multirow{2}{*}{ Number of experiments } & \multicolumn{3}{|c|}{ Subject A Male in his 30s } & \multicolumn{3}{|c|}{ Subject B Female in her 30s } & \multicolumn{3}{|c|}{ Subject C Female in her $60 \mathrm{~s}$} & \multicolumn{3}{|c|}{ Subject D Male in his $60 \mathrm{~s}$} \\
\hline & 1 & 2 & 3 & 1 & 2 & 3 & 1 & 2 & 3 & 1 & 2 & 3 \\
\hline Situation & Outing & 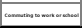 & Returning home & Returning home & Outing & 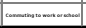 & Travel & Outing & Returning home & Returning home & 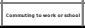 & Outing \\
\hline Means of transportation Result & Share Cycle & cab & $\mathrm{cab}$ & scheduled bus & walking & subway & Share Cycle & Share Cycle & Free circular bus & $\mathrm{cab}$ & Share Cycle & Share Cycle \\
\hline Facility Spot Genre Result No.1 & park & cafe & cafe & cafe & park & cafe & tourist attraction & park & massage & park & Shared Office & tourist attraction \\
\hline Facility Spot Genre Result No.2 & tourist attraction & massage & cafe & cafe & shopping mall & cafe & tourist attraction & park & tourist attraction & cafe & cafe & tourist attraction \\
\hline Facility Spot Genre Result No.5 & \begin{tabular}{|l} 
tourist attraction \\
\end{tabular} & sports gym & cafe & cafe & sports gym & cafe & tourist attraction & sports gym & massage & cafe & Shared Office & park \\
\hline
\end{tabular}

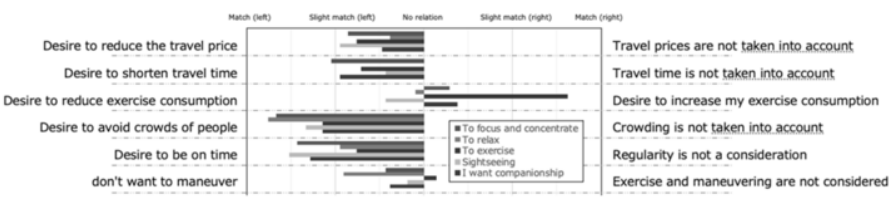

Figure 9 "Everyday intention" common data

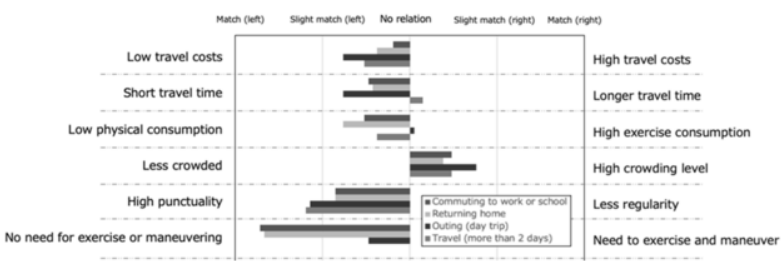

Figure 10 General data of "mobility situations"

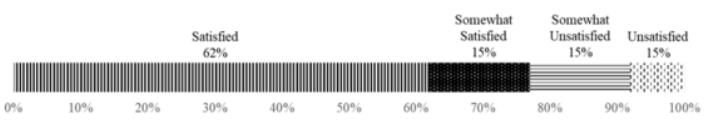

Figure 11 Means of mobility evaluation results

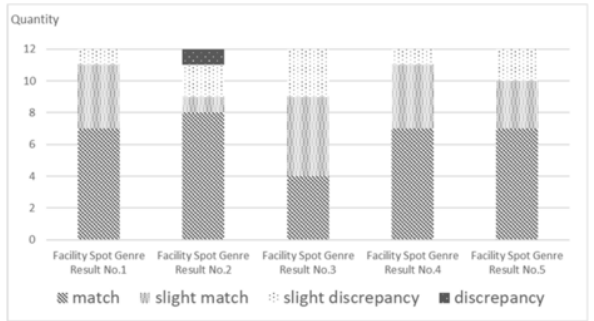

Figure 12 Activity spot evaluation results (Activity genres related to queries)

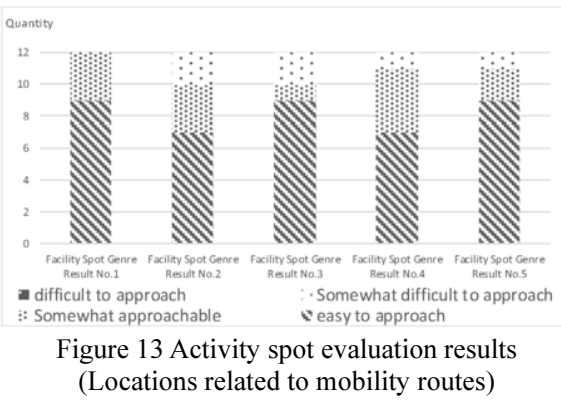




\subsection{Observations}

Based on the results of the coverage rate related to the search results, it is suggested that there is little polarization in the distribution of the data space. In particular, with regard to the means of mobility, the intention and situation context is reflected. If the types of mobility services increase moving forward, it is considered that the coverage rate will increase. For the facility spots, genres with low association were seen in some of the lower ranking spots. By filtering spots in the Rectangle Mobility Scope Model, the facility spots with a high similarity to the sensitivity to a context are selected in the mobility routes. Measures for improving this selection are related to the facility spot data quantity.

Next, in terms of the results of the fill-rate for queries, we show the extent to which sensitivity of mobile users can be expressed as queries. For items where a particular difference can be seen, it is considered that with a distance calculation this is an effective dimension/axis. Additionally, by applying general feature values in "everyday intention" and "mobility situation" vector data, this system realizes the generation of highly objective queries.

With regard to the result of the fill-rate for the output value, we have obtained a constant rating for both the means of mobility and the facility spot. Based on the results, the effectiveness of the series in the calculation methods including the query creation operators was shown. Additionally, some opinions were received from the test subjects. Favorable opinions included that "it was interesting how means of mobility and tourist sites I was not aware of were proposed", "going out has become more enjoyable", and "I want to use it when traveling to regions I don't know." On the other hand, improvement points were that there was a desire for personal and hobby-related factors matters to be reflected in the means of mobility, such as "being difficult to ride a bicycle due to being in one's 60s", and "when dedicating oneself to, or concentrating on, something, it is acceptable to take a longer way around a more coherent description of movement time." For matters related to means of activity, there was also a desire for psychological reassurance, stating that "I intend to be conducting the activity around the destination area before meeting somebody in that location" and "the activity method changes according to how long you have for the spare time". It was considered that more information was desirable, and it was stated that "they would like to know more about its feeling and what kind of things are imagined through photos and review information."

\section{Conclusion}

This paper presents a mobility and activity integration system for making routing plans for moving from an original point to a destination with a scenario as "sensitivity to context" on the route. The "sensitivity to context" expresses reactions to the intentions and situations of a moving user. This integration makes it possible to merge "mobility" and "activity" in temporal, spatial, and semantic ways, with concentrations on intellectual activities.

As the future outlook, we aim to make it possible to combine route searches with mobility, not only in the form of "one last mile", but also with free movement over a wider range. It will allow mobile users to select their appropriate routing from a variety of mobile services and mobility routes. Additionally, routing plans for activity could be flexible for supporting facility spots with disaster and event information (e.g.: blood donation, picking up garbage, roadside live music performances etc.). It will be related 
to achieving SDGs and constructing a platform supporting various issues.

\section{References}

[1] Nissay Asset Management Co., " $70 \%$ of the world's population in 2050 shall be city dwellers”, Market Report, May 2018.

[2] Ministry of Land, Infrastructure, Transport and Tourism, "Government/Private Enter prise Collaboration Platform for Smart Cities" https://www.mlit.go.jp/scpf/

[3] Katsuji Imada, Forum for a collaboration platform with pollution materials utilized i $\mathrm{n}$ SDGS through the power of citizens in Tokyo, Dec. 2018.

[4] METI : Study Group on New Mobility Services that can make IoT and AI possible - "Activation of new mobility services",https://www.meti.go.jp/shingikai/mono_info _service/smart_mobility_challenge/20190408_report.html

[5] Ministry of Land, Infrastructure, Transport and Tourism, Round-Table Discussion o $\mathrm{n}$ New Mobility Services for Urban and Regional Areas, "Overview of Interim Repo rt”, https://www.mlit.go.jp/sogoseisaku/transport/sosei_transport_tk_000089.html

[6] "Mobility as a Service (MaaS) constitutes the integration of various forms of transport services into a single mobility service accessible on demand," MaaS Alliance White Paper, MaaS Alliance, 2017.

[7] Yasushi Kiyoki, Takashi Kitagawa, Takanari Hayama: "A meta-database system for semantic image search by a mathematical model of meaning," ACM SIGMOD Record, Vol.23 Issue4, December 1994.

[8] Yasuhiro Hayashi, Yasushi Kiyoki, and Xing Chen. "A Combined Image-Query Cre ation Method for Expressing User's Intentions with Shape and Color Features in $\mathrm{Mu}$ ltiple Digital Images," Information Modelling and Knowledge Bases (IOS Press), p p.258-277, Vol. XXII, March 2011.

[9] Yasuhiro Hayashi, Yasushi Kiyoki, and Xing Chen, "An Image-Query Creation Met hod for Expressing User's Intentions by Combining Multiple Images," Information Modelling and Knowledge Bases (IOS Press), pp.188-207, Vol. XXI, March 2010.

[10] Atsushi Tsuya, Creating diverse driving plans using sensitivity rating results of pe ople's motives when traveling, 2011, Vol. 10, No.3 p. 433-443.

[11] Cabinet Public Relations Office : Free time and tourism, Monthly Public Opinion Survey, No.11, 2004, pp.3-84.

[12] Google Maps, https://www.google.co.jp/maps/.

[13] Jalan Tourism Guide, https://www.jalan.net/travel/.

[14] Trip Advisor, https://www.tripadvisor.jp/.

[15] Syuichi Kurabayashi, Naoki Ishibashi and Yaushi Kiyoki, "A Multidatabase Syste $\mathrm{m}$ Architecture for Integrating Heterogeneous Databases with Meta-Level Active Ru le Primitives", Proceedings of the 20th IASTED International Conference on Applie d Informatics, pp478-387, 2002.

[16] OpenWeatherMap, https://openweathermap.org/.

[17] JapanHoliday, https:/github.com/suzuki-shunsuke/japanese-holiday-api.

[18] Google Maps Platform, https://cloud.google.com/maps-platform/. 